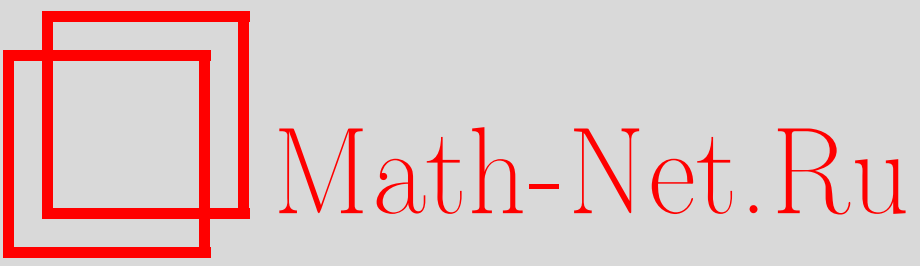

В. С. Новиков, Уравнения, инвариантные относительно дифференциальных подстановок, ТМФ, 1998, том 116, номер 2, 193-200

DOI: https://doi.org/10.4213/tmf897

Использование Общероссийского математического портала Math-Net.Ru подразумевает, что вы прочитали и согласны с пользовательским соглашением

http://www.mathnet.ru/rus/agreement

Параметры загрузки:

IP: 52.205.19.152

26 апреля 2023 г., 15:03:48 
ТЕОРЕТИЧЕСКАЯ

И МАТЕМАТИЧЕСКАЯ

ФИЗИКА

Том 116, № 2

август, 1998

(C) 1998 г.

В. С. Новиков*

\section{УРАВНЕНИЯ, ИНВАРИАНТНЫЕ ОТНОСИТЕЛЬНО ДИФФЕРЕНЦИАЛЬНЫХ ПОДСТАНОВОК}

Рассматривается задача классификации дифференциальных автоподстановок вида $\hat{u}=G\left(u_{x}, u\right)$, допускаемых эволюционными уравнениями общего вида с одной пространственной переменной. Показано, что в однополевом случае любая такая подстановка сводится точечным преобразованием к автоподстановке $\hat{u}=u+u_{x} / u$ для уравнения Бюргерса. Приведены обобщения этой подстановки на двухполевой случай.

\section{ВВЕДЕНИЕ}

Рассмотрим скалярное эволюционное уравнение с одной пространственной переменной

$$
\begin{gathered}
u_{t}=F\left(u, u_{x}, u_{x x}, \ldots, u^{(l)}\right), \\
u^{(l)}=\frac{\partial^{l} u}{\partial x^{l}}
\end{gathered}
$$

где $l$ - порядок уравнения, допускаюшее автоподстановку (преобразование Бэклунда) вида

$$
\hat{u}=G\left(u_{x}, u\right)
$$

Задача о классификации преобразований Бэклунда, допускаемых эволюционными уравнениями второго порядка, описана в недавней работе [1]. В отличие от этой работы мы фиксируем достаточно простой вид автопреобразования, но зато снимаем ограничения на класс рассматриваемых уравнений. Другое важное отличие данной работы состоит в том, что изучаемые преобразования Бэклунда являются вырожденными и характерны для уравнений типа $\mathrm{C}$, а не $\mathrm{S}$ в терминологии Калоджеро [2]. Классический пример - уравнение Бюргерса

$$
u_{t}=u_{x x}+2 u u_{x}
$$

допускающее автоподстановку

$$
\hat{u}=u+\frac{u_{x}}{u}
$$

${ }^{*}$ Институт теоретической физики им. Л. Д. Ландау РАН, Москва, Россия 
и линеаризуемое преобразованием Коула-Хопфа $u=v_{x} / v$. Кроме того, ясно, что линейные уравнения с постоянными коэффициентами обладают богатым набором дискретных симметрий вида (2). В [1] было показано, что для уравнений второго порядка с точностью до точечных замен $u=\phi(\tilde{u})$ имеются лишь две автоподстановки вида (2): преобразование Бэклунда (3) для уравнения Бюргерса и подстановка $\hat{u}=c_{1} u_{x}+c_{2} u$ для линейного уравнения с постоянными коэффициентами. Основным результатом этой работы является обобщение этого утверждения на уравнения произвольного порядка.

В недавней работе [3] рассматривалась классификация подстановок вида $v=$ $P\left(x, u, u_{x}\right)$, которые переводят решения некоторого эволюционного уравнения в решения другого эволюционного уравнения. Было показано, что существует чрезвычайно широкий произвол как в выборе дифференциальных подстановок, так и уравнений, их допускающих. В нашей работе показано, что такой произвол изчезает при рассмотрении автоподстановок.

\section{1. СКАЛЯРНЫЕ ЭВОЛЮЩИОННЫЕ УРАВНЕНИЯ}

Будем называть автоподстановки вида (2), а также уравнения (1), их допускающие, эквивалентными, если они переводятся друг в друга точечными заменами $u=\phi(\tilde{u})$ и растяжениями координат $x \rightarrow \alpha x, t \rightarrow \beta t$. Сформулируем основной результат данной работы.

ТЕОРЕма 1. Любая дифференциальная подстановка вида (2) әквивалентна одной из следующих:

$$
\begin{aligned}
& \hat{u}=u+\frac{u_{x}}{u}, \\
& \hat{u}=c_{1} u_{x}+c_{2} u,
\end{aligned}
$$

а уравнения, их допускающ,е, - соответственно иерархия уравнений Бюргерса

$$
u_{t}=R^{l-1}\left(u_{x}\right)
$$

где $R$ - оператор рекурсии

$$
R=D_{x}+u+u_{x} D_{x}^{-1}
$$

и линейное уравнение с постоянными коэффициентами

$$
u_{t}=u^{(l)}+\sum_{k=0}^{l-1} a_{k} D_{x}^{k} u .
$$


ДокАЗАТЕЛЬСтво. Прежде всего заметим, что определение иерархии уравнений Бюргерса (6) корректно, т.к. при любом $l>1$ правая часть (6) есть полная производная по $x$. Перепишем формулу (2) в виде

$$
u_{x}=A\left(u_{1}, u\right)
$$

Согласно формуле (8) мы можем переходить от динамических переменных $u, u_{x}, u_{x x}, \ldots$ $\ldots, u^{(l)}$ к динамическим переменным $u, u_{1}, u_{2}, \ldots$ Для того чтобы уравнение (1) было инвариантно относительно подстановки (8), необходимо выполнение условия совместности

$$
D_{x} F=D_{t} A
$$

Удобно линеаризовать уравнение $(9)$, а именно ввести операторы $F_{*}$ и $A_{*}$ по формуле $[4,5]$

$$
f_{*}(v)=\left.\frac{\partial}{\partial \varepsilon} f\left(u+\varepsilon v, u_{x}+\varepsilon v_{x}, \ldots\right)\right|_{\varepsilon=0},
$$

тогда

$$
A_{*}=A_{1} T+A_{0}, \quad F_{*}=\sum_{k=0}^{l} F_{k} D_{x}^{k},
$$

где

$$
\begin{aligned}
A_{i} & \equiv \frac{\partial}{\partial u_{i}} A \equiv \partial_{i} A, \\
F_{i} & \equiv \frac{\partial}{\partial u^{(i)}} F
\end{aligned}
$$

а $T$ - оператор сдвига динамических переменных $T: u \rightarrow u_{1}$. Линеаризуем теперь (9) и исключим производные по $t$ согласно (1):

$$
\begin{aligned}
\left(D_{x} F\right)_{*}(v)= & \sum_{k=0}^{l} \sum_{j=0}^{l} F_{k, j} u^{(k+1)} D_{x}^{j}(v)+\sum_{k=0}^{l} F_{k} D_{x}^{k+1}(v)= \\
= & \left(D_{t} A\right)_{*}(v)=A_{1,1} T(F) v_{1}+A_{1,0} T(F) v+A_{1} \sum_{k=0}^{l} T\left(F_{k}\right) D_{x}^{k}\left(v_{1}\right)+ \\
& +A_{1,0} F v_{1}+A_{0,0} F v+A_{0} \sum_{k=0}^{l} F_{k} D_{x}^{k}(v) .
\end{aligned}
$$

Воспользуемся связью

$$
v_{x}=A_{1} v_{1}+A_{0} v \Longrightarrow v_{1}=\frac{1}{A_{1}} D_{x}(v)-\frac{A_{0}}{A_{1}} v
$$

и исключим $v_{1}$ из $(10)$. Тогда, приравняв нулю коэффициенты при каждой степени выражения $D_{x}^{i}(v), i=0,1, \ldots, l+1$, получим систему уравнений, из которой определим вид функций $A$ и $F$. При $D_{x}^{l+1}(v)$ имеем

$$
F_{l}=T\left(F_{l}\right)
$$


Из этого уравнения следует, что $F_{l}$ является константой. Очевидно, что за счет растяжения времени можно, не теряя обшности, положить $F_{l}=1$. Подставив $F_{l}=1$ в $(10)$, получим при $D_{x}^{l}(v)$

$$
F_{l-1}=T\left(F_{l-1}\right)+l A_{1} D_{x}\left(\frac{1}{A_{1}}\right) .
$$

Покажем, что это уравнение имеет следующие решения:

$$
F_{l-1}=l u+\text { const }, \quad A=u\left(u_{1}-u\right)
$$

и

$$
F_{l-1}=\text { const }=c_{1}, \quad A=u_{1}+\phi(u) .
$$

Теперь удобно перейти к переменным $u, u_{1}, u_{2}, \ldots, u_{l-1}$ :

$$
u_{x}=A\left(u_{1}, u\right), \quad u_{x x}=D_{x}\left(A\left(u_{1}, u\right)\right), \quad \ldots, \quad u_{1, x}=A\left(u_{2}, u_{1}\right), \quad \ldots
$$

Тогда уравнение (11) перепишется в виде

$$
l D_{x} \log A_{1}=(T-1)(f), \quad f \equiv F_{l-1} .
$$

Перепишем уравнение (14), раскрывая производную логарифма и заменяя производные $u_{x}$ и $u_{1, x}$ согласно формуле $(2)$ на $A$ и $T(A)$, соответственно, в виде

$$
l \frac{A_{1,1}}{A_{1}} T(A)+l \frac{A_{1,0}}{A_{1}} A=(T-1)(f) .
$$

Легко видеть, что $f=f\left(u_{1}, u\right)$. Дифференцирование $(15)$ по $u_{2}$ дает

$$
l \frac{A_{1,1}}{A_{1}} T\left(A_{1}\right)=T\left(f_{1}\right), \quad f_{i}=\frac{\partial}{\partial u_{i}} f=\partial_{i} f,
$$

откуда

$$
l \frac{A_{1,1}}{A_{1}}=T\left(\frac{f_{1}}{A_{1}}\right) .
$$

Дифференцируя это выражение по $u$, получаем

$$
\partial_{0} \frac{A_{1,1}}{A_{1}}=0 \quad \Longrightarrow \quad A_{1,1}=\alpha\left(u_{1}\right) A_{1} .
$$

С помощью соотношения (17) легко получить, что общий вид $A$ есть

$$
A=a\left(u_{1}\right) b(u)+c(u),
$$

причем $a \neq$ const. Покажем, что калибровочным преобразованием можно добиться того, что $a\left(u_{1}\right)=u_{1}$. Действительно, из (16) следует, что

$$
f=u_{x} \alpha(u)+\delta(u)
$$


Уравнение (1), следовательно, имеет вид

$$
u_{t}=u^{(l)}+\left(u_{x} \alpha(u)+\delta(u)\right) u^{(l-1)}+\widetilde{F}\left(u^{(l-2)}, \ldots, u\right) .
$$

С делаем калибровочное преобразование $u=\phi(\hat{u})$. Тогда уравнение $(18)$ перепишется в виде

$$
\begin{aligned}
\hat{u}_{t}= & \hat{u}^{(l)}+\hat{u}^{(l-1)} \hat{u}_{x}\left(\phi_{\hat{u}}(\hat{u}) \alpha(\phi(\hat{u}))+2(l-1) \frac{\phi_{\hat{u} \hat{u}}(\hat{u})}{\phi_{\hat{u}}(\hat{u})}\right)+ \\
& +\delta(\phi(\hat{u})) \hat{u}^{(l-1)}+\widehat{F}\left(\hat{u}^{(l-2)}, \ldots, \hat{u}\right) .
\end{aligned}
$$

Пусть функция $\phi(\hat{u})$ удовлетворяет уравнению

$$
2(l-1) \phi_{\hat{u} \hat{u}}(\hat{u})+\phi_{\hat{u}}^{2}(\hat{u}) \alpha(\phi(\hat{u}))=0 .
$$

Решение этого уравнения дается неявной формулой

$$
\hat{u}=\int \exp \left[\frac{g(t)}{2(l-1)}\right] d t,
$$

где

$$
g=\int \alpha(t) d t
$$

т.e.

$$
A=\gamma(u) u_{1}+\beta(u) .
$$

Возможны два случая: $\gamma(u) \neq$ const и $\gamma(u)=$ const. Рассмотрим первый случай. Подставив (19) в (15), получаем

$$
l \frac{\gamma^{\prime}(u)}{\gamma(u)}\left(u_{1} \gamma(u)+\beta(u)\right)=(T-1)(f) .
$$

Легко видеть, что $f=f(u)$. Дифференцируя $(20)$ по $u_{1}$, получаем уравнения

$$
f^{\prime}\left(u_{1}\right)=l \gamma^{\prime}(u)=\mathrm{const}=c \quad \Longrightarrow \quad \gamma(u)=\frac{c}{l} u+c_{1}, \quad f(u)=c u+c_{2},
$$

которые подставляем в (20), и находим

$$
\beta(u)=-\frac{c}{l} u^{2}-c_{1} u
$$

Итак, с точностью до калибровочного преобразования и растяжения координат мы получили решение (12). Второй случай, очевидно, дает решение (13).

Легко показать, что решение (13) приводит к линейному уравнению с постоянными коэфрициентами.

При заданной функции $A$ из уравнения (10) однозначно восстанавливается функция $F$. Хорошо известно, что уравнения Бюргерса (6) коммутируют с подстановкой (4). Следовательно, учитывая формулы (12) и (13), мы таким образом находим все решения уравнения (10). Теорема 1 доказана.

Аналогично доказывается следующая теорема. 
ТЕОрема 2. Уравнение (1) допускает автоподстановку (2) тогда и только тогда, когда оно сводится $к$ линейному некоторой подстановкой вида

$$
u=H\left(v, v_{x}\right)
$$

В заключение отметим, что не всякое уравнение класса С вида

$$
u_{t}=F\left(x, u, u_{x}, u_{x x}, \ldots\right)
$$

обладает некоторой нетривиальной дискретной симметрией

$$
\hat{u}=G\left(x, u, u_{x}\right) .
$$

Например, уравнение

$$
u_{t}=u^{2} u_{x x}
$$

сводится к линейному $\hat{u}_{t}=\hat{u}_{\hat{x} \hat{x}}$ заменой

$$
d \hat{x}=u^{-1} d x-u_{x} d t, \quad \hat{u}=u,
$$

но не обладает дискретной симметрией вида (21).

\section{2. СИСТЕМЫ УРАВНЕНИЙ ВТОРОГО ПОРЯДКА. ПРИМЕРЫ}

Рассмотрим системы второго порядка вида

$$
\begin{aligned}
& u_{t}=F\left(u_{x x}, v_{x x}, u_{x}, v_{x}, u, v\right), \\
& v_{t}=G\left(u_{x x}, v_{x x}, u_{x}, v_{x}, u, v\right) .
\end{aligned}
$$

Для таких систем можно поставить аналогичную задачу, а именно описать с точностью до калибровочного преобразования $u=\phi(\tilde{u}, \tilde{v}), v=\psi(\tilde{u}, \tilde{v})$ все такие системы, инвариантные относительно автопреобразования Бэклунда,

$$
u_{x}=A\left(u_{1}, u, v\right), \quad v_{x}=B\left(v_{1}, u, v\right) .
$$

Такую задачу можно полностью решить методом, описанным в разделе 1 . Мы не будем здесь подробно на этом останавливаться и приведем два примера.

Система

$$
\begin{aligned}
u_{t}= & u_{x x}+2 u u_{x}+2 v u_{x}-(\alpha-1) u v_{x}+2 \beta u_{x}+\gamma v_{x}-(\alpha-1) u^{2} v-(\alpha-1) v^{2} u+ \\
& +2(\beta-\eta) u v-\delta u^{2}+\gamma v^{2}+\mu u+\nu v, \\
v_{t}= & \alpha v_{x x}+2 \alpha v v_{x}+2 \alpha u v_{x}+(\alpha-1) v u_{x}+2 \eta v_{x}+\delta u_{x}+(\alpha-1) u^{2} v+(\alpha-1) v^{2} u- \\
& \quad-\gamma v^{2}+\delta u^{2}-2(\beta-\eta) u v-\mu u-\nu v
\end{aligned}
$$

обладает преобразованием Бэклунда

$$
u_{x}=\left(u_{1}-u\right)(u+v+\kappa), \quad v_{x}=\left(v_{1}-v\right)(u+v+\kappa)
$$


(здесь греческими буквами обозначены произвольные константы). Эта система сводится к линейной

$$
\begin{aligned}
& p_{t}=p_{x x}+2 \beta p_{x}-\gamma q_{x}+\rho p-\nu q+\omega \\
& q_{t}=\alpha q_{x x}-\delta p_{x}+2 \eta q_{x}+\mu p+(\rho-\mu-\nu) q+\omega
\end{aligned}
$$

подстановкой

$$
u=\frac{p_{x}}{p-q}, \quad v=-\frac{q_{x}}{p-q},
$$

найденной В. Г. Марихиным.

Система

$$
\begin{aligned}
& u_{t}=u_{x x}+2 u u_{x}+2 v u_{x}+2 \beta u_{x}+(\beta-\gamma) v_{x}+\delta(u+v)^{2}+\mu(u+v), \\
& v_{t}=v_{x x}+2 v v_{x}+2 u v_{x}+2 \gamma v_{x}-(\beta-\gamma) u_{x}-\delta(u+v)^{2}-\mu(u+v)
\end{aligned}
$$

обладает преобразованием Бэклунда

$$
\begin{aligned}
u_{x}= & \left(u_{1}-u\right)(u+v+\alpha)+(\beta-\gamma+\alpha+\eta-\delta)(u+v)+ \\
& +(\delta-\beta+\gamma)(u+v+\alpha) \log (u+v+\alpha)+\nu \\
v_{x}= & \left(v_{1}-v\right)(u+v+\alpha)+(\delta-\beta+\gamma-\alpha-\eta)(u+v)- \\
& -(\delta-\beta+\gamma)(u+v+\alpha) \log (u+v+\alpha)-\nu
\end{aligned}
$$

и сводится к линейной

$$
\begin{aligned}
& p_{t}=p_{x x}+2 \beta p_{x}+(\gamma-\beta) q_{x}+\mu p-\mu q+\rho, \\
& q_{t}=q_{x x}+(\beta-\gamma) p_{x}+2 \gamma q_{x}+\mu p-\mu q+\rho
\end{aligned}
$$

подстановкой

$$
\begin{aligned}
& u=\frac{p_{x}}{p-q}+(\beta-\gamma-\delta) \log (p-q)+(\gamma+\delta-\beta+\xi)+\frac{\zeta}{p-q} \\
& v=\frac{q_{x}}{q-p}-(\beta-\gamma-\delta) \log (p-q)-(\gamma+\delta-\beta+\xi)-\frac{\zeta}{p-q}
\end{aligned}
$$

Можно показать, что автоподстановки вида (23) сводятся точечными преобразованиями к виду

$$
u_{x}=u_{1} \alpha(u, v)+\beta(u, v), \quad v_{x}=v_{1} \gamma(u, v)+\delta(u, v)
$$

По-видимому, справедливо также утверждение, что система (22) обладает преобразованием Бэклунда (23) тогда и только тогда, когда эта система линеаризуема.

Автор выражает благодарность своему научному руководителю профессору А.Б. Шабату, поставившему эту задачу и внесшему ряд сушественных замечаний в метод и текст статьи, а также В.Г. Марихину за ряд ценных частных сообщений. 


\section{Список литературы}

[1] В. Э. Адлер, А. Б. Шабат. ТМФ. 1997. Т. 111. С. 323.

[2] What Is Integrability? Ed. V.E. Zakharov. Berlin-Heidelberg: Springer-Verlag, 1991.

[3] C. Я. Старцев. Дифференциальные подстановки и симметрии гиперболических уравнений. В кн.: Асимптотики и симметрии в нелинейных динамических системах. Уфа: ИМ с ВЦ РАН, 1995. C. $80-95$.

[4] П. Олвер. Приложения групп Ли к дифференциальным уравнениям. М.: Мир, 1989.

[5] А. В. Михайлов, А. Б. ШШабат, Р. И. Ямилов. УМН. 1987. Т. 42. № 4. С. 3.

Поступила в редакцию5 5.III.98 г. 